\title{
Evaluation of a Behind-the-Ear ECG Device for Smartphone based Integrated Multiple Smart Sensor System in Health Applications
}

\author{
Numan Celik ${ }^{1 *}$, Nadarajah Manivannan ${ }^{1}$, Wamadeva Balachandran ${ }^{1}$ \\ ${ }^{1}$ Department of Computer and Electronics Engineering \\ Brunel University London \\ London, United Kingdom
}

\begin{abstract}
In this paper, we present a wireless Multiple Smart Sensor System (MSSS) in conjunction with a smartphone to enable an unobtrusive monitoring of electrocardiogram (ear-lead ECG) integrated with multiple sensor system which includes core body temperature and blood oxygen saturation $\left(\mathrm{SpO}_{2}\right)$ for ambulatory patients. The proposed behind-the-ear device makes the system desirable to measure ECG data: technically less complex, physically attached to non-hair regions, hence more suitable for long term use, and user friendly as no need to undress the top garment. The proposed smart sensor device is similar to the hearing aid device and is wirelessly connected to a smartphone for physiological data transmission and displaying. This device not only gives access to the core temperature and ECG from the ear, but also the device can be controlled (removed and reapplied) by the patient at any time, thus increasing the usability of personal healthcare applications. A number of combination ECG electrodes, which are based on the area of the electrode and dry/non-dry nature of the surface of the electrodes are tested at various locations near behind the ear. The best ECG electrode is then chosen based on the Signal-toNoise Ratio (SNR) of the measured ECG signals. These electrodes showed acceptable SNR ratio of $\sim 20 \mathrm{db}$, which is comparable with existing tradition ECG electrodes. The developed ECG electrode systems is then integrated with commercially available PPG sensor (Amperor pulse oximeter) and core body temperature sensor (MLX90614) using a specialized micro controller (Arduino UNO) and the results monitored using a newly developed smartphone (android) application.
\end{abstract}

Keywords—wireless body area networks; body-worn sensors; ECG; core body temperature; oxygen saturation level $\left(\mathrm{SpO}_{2}\right)$; biosensor integration; $m$-health

\section{INTRODUCTION}

The rapid growth of wireless technologies brings new innovative ideas that enables continuous real-time remote patient monitoring in healthcare services using compact wireless body sensors. The services and technologies provide relatively uncontroversial, well-communicated and monitoring devices, developed to give more affordable solutions specifically for mobile healthcare, such as daily activity monitoring, personal healthcare and monitoring systems, and body sensor systems that can alert the clinicians via the patients' mobile phones. New trend in remote patient monitoring is moving toward the use of personal mobile devices compatible with multiple biomedical sensors using wireless communication, such as Bluetooth and Zigbee [1].

In practice, this recent mobile health (m-Health) technology enables to see the people's daily activity in their smartphones. In addition, these mobile-based portable embedded devices will provide platforms to monitor their critical physiological data continuously and remotely. An assessment report has been prepared for the European Union regarding the effectiveness of $\mathrm{m}$-Health in biomedical applications and the diagnosis of the diseases in 2013. According to this report, m-Health applications could save $€ 99$ billion in healthcare costs in the EU and add $€ 93$ billion to the EU GDP in 2017, if its adoption is encouraged [2].

An integrated wearable monitoring system, which aim to bring compact body sensors such as electrocardiography (ECG), blood pressure, photoplethysmography (PPG), core body temperature (CBT), heart rate, pulse oximetry, and EEG together forms the concept of a wireless body area network (WBAN) or personal area network (PAN) and displays the physiological signals on a monitoring device. One of the benefits that PAN systems bring is the ability to integrate multiple intelligent sensors, wireless connectivity and a battery into a wearable patch unit that sends the physiological data to a mobile device. Figure 1 indicates the concept of a typical WBAN where the general tasks of the electronics designer are compactness, integration of body sensors and wireless connectivity, including a telemedicine system, which can alert a clinician when life-threatening changes occur or to provide a feedback to the patient to help maintain an optimal health status using Cloud health services. 


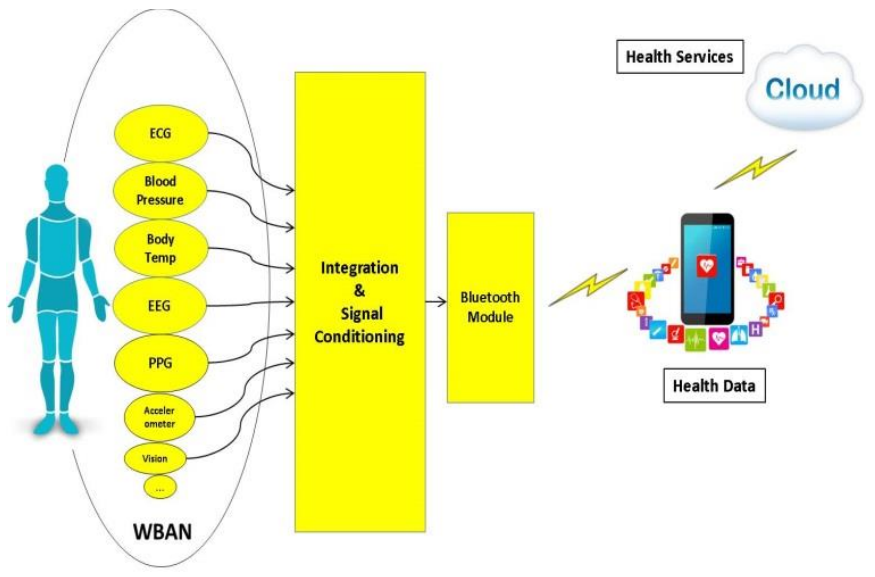

Fig. 1. Typical personal health system with integrated sensor network (wireless body area network)

Here, we examine a continuous, wearable and wireless critical-sign patient monitoring system which was focused on integrating ECG sensors placed behind the ear; CBT sensor placed in the ear; and PPG sensor clipped on the finger. The reason for choosing the ear as a location in these experiments is that it makes possible to measure ECG and CBT together with subject comfortless of ECG monitoring. Behind the ear region has less hair than chest and non-hair regions give more suitability for long term use of ECG monitoring. Moreover, the preparation of skin will not be a necessity and it will not be difficult to remove the sensors that bring a user-friendly perspective to the patients and clinicians.

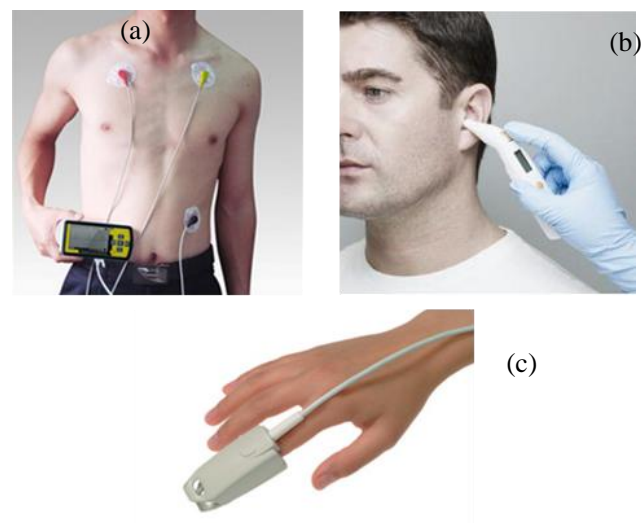

Fig. 2. (a) the typical 3-lead ECG monitoring system; (b) ear-tympanic device to measure core body temperature; (c) the reflective PPG sensor to measure blood oxygen saturation $(\mathrm{SpO} 2)$ and heart rate

This paper aims to advance the ambulatory ECG treatments by proposing a wearable behind-the-ear smart sensor that transmits integrated physiological data wirelessly to a smartphone and monitors ECG, CBT, heart rate and $\mathrm{SpO}_{2}$. Since most conventional systems take each data separately and from different locations on the body (see in Figure 2), the proposed work based on ear-lead ECG monitoring integrated with the multisensory system is important for many reasons. Firstly, this is the first attempt of ear-lead ECG monitoring to be integrated with two other body-sensors, PPG and tympanic sensors to measure heart rate, $\mathrm{SpO}_{2}$ and $\mathrm{CBT}$ respectively. Secondly, a smart sensor system is developed that facilitates sensor integration by sealing into an ear mold. Thirdly, the collected health data in the microcontroller unit (MCU) are wirelessly transferred to an Android smartphone and an application is written to display ECG, CBT and PPG data. Another aim of this paper was to investigate ECG signals when different types of electrodes (gel and dry electrodes) have been placed on different locations on the body, such as chest, ear and arm regions. Moreover, this design is less disrupted by motion artifacts for ear-lead ECG monitoring when compared to the previous work which is done by Da He [27] using two ECG sensors. In the future, this option not only gives flexibility to the patient, but also can give the clinicians to access the physiological data in real time via online access from personal healthcare records.

The remainder of this paper is organized as follows: Section 2 includes related works on this topic and deals with the problem formulation; Section 3 explains the system description of the work; Section 4 illustrates experimental results and analysis of ear-lead ECG using different sensors onto the different locations of the body; and also the demonstrations of CBT and PPG sensors. Section 4 also draws the combining ECG sensing unit with the integrated CBT and PPG sensors, and demonstrates wireless transmission between integrated MCU unit and a smartphone; finally conclusion and discussion are presented in Section 5.

\section{RELATED WORK}

When a ubiquitous smart sensor is developed, there should be basic components that should be considered: easy-to-use, mobility, accuracy, and security. There are currently many ongoing researches that investigate various solutions on the design of wireless personal healthcare monitoring devices [35]. Lee et al. developed a mobile phone based ECG monitoring application. The system describes the design and implementation of a prototype tele-health system which monitored physiological signals of patients in real-time [4]. Sanches et al. designed an electronic temperature sensor within a headset Bluetooth device that sends the temperature measurements to a mobile phone. The proposed system measures central body temperature continuously at the ear [6]. Jung et al. proposed a wireless body sensor platform called 'Virtual Cuff' that comprises PPG and ECG sensors to estimate systolic and diastolic blood pressure (BP). The presented work fuses data from various sensors, including ECG, PPG, accelerometer, and GPS, for extrapolating BP information using signal characteristics that are derived from PPG and ECG waveforms [7]. Do Valle et al. examined [8] a behind-the-ear device that records EEG measurements on smartphone continuously and then uploads the patients' data to a secure server. Song et al. developed a body monitoring system design based on android smartphone including three main functions such as brainwave capture (EEG), ECG and temperature. These data are gathered by hardware and sent to the Bluetooth receiving device of android smartphone [9]. Boano et al. managed to measure core body temperature (CBT) on ambulatory patients and exercising athletes using a wireless wearable device that measures the tympanic temperature at the ear. The CBT data is transmitted via ATmega128RFA1 chip based on ZigBee communication which is different from other studies [10]. 
The authors of [11] proposed an ultra-wearable smart sensor system which combines ECG, tri-axial accelerometer, and GPS sensors to measure normal or elderly person's daily activities. This device also encompasses voice biofeedback and data fusion technologies in order to accommodate future needs and to make the smart sensor much better. The hardware unit of the system consists of a TI MSP430 microprocessor, Bluetooth wireless transmission to PC client software, micro SD card storage, and LCD display. The embedded algorithm combines two sensors for noise reduction, and utilizes voice biofeedback for exercise overload warning. Hernandez et al. demonstrated [12] that motion sensors of a smartphone can be used to recover heart and breathing rates of the users during stationary positions and activities while the smartphone was being carried in a bag or pocket or even during listening on the phone. They developed these rates from accelerometer data and compared them with measurements obtained with FDA-cleared sensors by evaluating effective accuracy numbers. Hii et al. presented [13] a comprehensive ubiquitous healthcare solution which includes a real time ECG monitoring and analyzing system based on an Android mobile device and also provides medicine care assistance. Wireless sensor network (WSN) technology is used in this system in order to transmit ECG data wirelessly from the patient's body to a smartphone device. As for medicine care assistance, barcode technology is applied to assist out-patients in medication administration, by capturing and decoding the barcode on medicines using the smartphone's embedded camera.

Wahl and his co-workers designed [15] an eyeglass (WISEglass) that consists of inertial motion, environmental light, and pulse sensors, processing and wireless data transmission functionality and also a rechargeable battery. The users will be able to monitor their daily activity recognition, screen-use detection, and heart rate estimation, because of having accelerometer, gyroscope and pulse sensors in itself. Regarding the work in ear-worn devices, the authors in [16] attempted to develop probes for Heart-phone to make such an unobtrusive earphone to measure heart rate using PPG technology. According to their design, the reflective photo sensor is embedded into each earbud on a pair of regular earphones. To obtain measurements, the sensor earphones are inserted into the ear and positioned such that the reflective photo sensor is against the inner side of ear. Then they can measure the amount of the reflected light from the blood vessels in the region. Moron et al. studied [17] on the technical performance of medical wireless personal area network (WPANs) that are based on smartphones. According to their telemedicine prototype, an Android based smartphone acts as a gateway between a set of wireless medical sensors and a data server. They also wanted to see the differences while modifying the smartphone model, the type of sensors connected to the WPAN, the use of other peripherals such as GPS receiver, the impact of the use of the Wi-Fi interface. The authors of [18] presented a wearable monitoring system to measure the driver alertness, evaluated by a smartwatch device based on fusion of direct and indirect method. The driver chronic physiological state is monitored by adopting a PPG sensor on the driver's finger that is connected to a wrist-type wearable device. A Bluetooth low energy module connected to the wearable device transmits the PPG data to the smartwatch in real-time. Lin et al. [20] proposed a wearable PPG sensor module based on a Programmable System on a Chip (PSoC), in the course of driving. It transmits measured PPG signal from earlobe to a smartphone via Bluetooth. On the smartphone, a heart rate $(\mathrm{HR})$ detection algorithm is implemented. When the abnormal HR is detected, the smartphone uses the sound and vibration to warn the driver using a magnetic ring. At the same time, physiological data and GPS location are also be transmitted to a data server (healthcare server system) via the $3 \mathrm{G}$ mobile network, so that the staff in the server system can monitor the recent information and monitor the driver's status.

The brief summary of this literature review finds the use of the modern communication technology for data exchange between ambulatory patients and mobile devices by combining several sensors in one typical system. This is one of the key factors to make m-Health platform much more desirable. However, conventional ECG monitoring systems, mentioned in the literature work, are measured from the chest and require more than 3 sensors in some cases. While trying to get ECG data from the chest, it is an undesirable method to integrate ECG sensors with other multiple sensors on the body, causing complexity for the whole system. This complexity would increase the noise level of each sensor and also the power requirement for the system. In the proposed sensor system, we are focusing the combination of an ear-lead ECG monitoring with integrated CBT and PPG multisensory system for mhealth applications. In order to avoid complexities, ear-lead ECG method was used to avoid hairy region and easy-to -use perspectives. The proposed $\mathrm{m}$-health application is not only a solution for the complexities involved in traditional of ECG monitoring system, but also enables an integrated and personalized smart device.

\section{SYSTEM DESCRIPTION}

The smart sensor platform was developed to experimentally combine three different body sensors and monitor regarded vital signals on the smartphone. The three sensors of the proposed system are ECG with behind the ear electrode, core body temperature (tympanic sensor in the ear canal), and both heart rate and blood oxygen saturation (PPG from the finger), and then the detected signals are communicated to a mobile client by using Bluetooth connection. The ECG and CBT sensors for displaying the physiological data are integrated in the form of an earbud. PPG sensor was attached onto the finger and connected to the microcontroller unit (MCU). Afterwards, the physiological signals are monitored on the mobile phone. Figure 3 shows the block diagram of the smart sensor system.

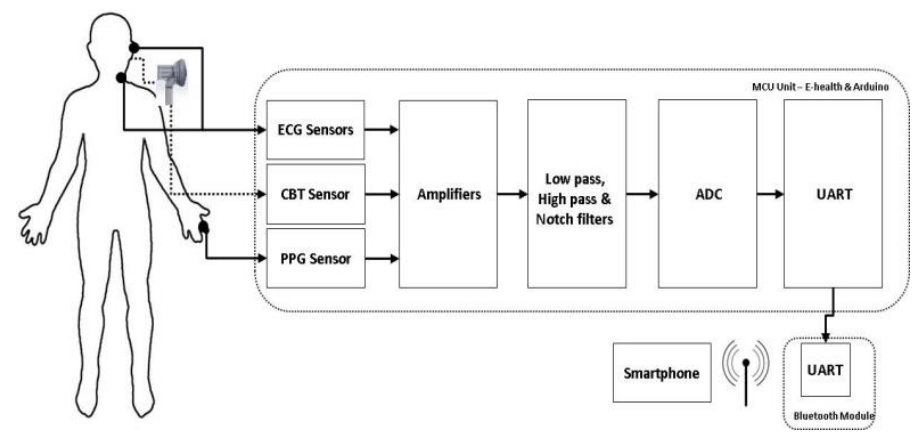

Fig. 3. System block diagram 


\section{A. ECG Sensing Unit}

As can be seen from the block diagram, ECG and CBT sensors were deployed together because of leading sensors near the ear location, but PPG sensor is clipped onto the finger. Three ECG sensors in different sizes were used and tested using both gel and dry electrodes. Table 1 lists the properties of three commercially available electrodes, which were used in the experiments. In the proposed system, the first electrode of three-electrode setup was placed behind the ear; the second electrode was attached on the upper neck area, and the last electrode was placed on the arm. Initially, two types of gel (adhesive $\mathrm{Ag}-\mathrm{AgCl}$ ) electrodes were used to get ECG data from the ear. One of the gel electrodes was known as 'Covidien electrode', which has a diameter of $24 \mathrm{~mm}$, and the second electrode was slightly bigger, which has a diameter of $38 \mathrm{~mm}$. Both electrodes are of $1 \mathrm{~mm}$ of thickness. After using these two gel electrodes, a number of dry electrodes were applied to the skin to detect ECG signals. The dimensions of this dry electrode are $10 \mathrm{~mm}$ (diameter) and $2 \mathrm{~mm}$ (thickness). Figure 4 shows the photographs of used electrodes.

TABLE I. THE PROPERTIES OF ELECTRODES USED FOR THE EXPERIMENTS

\begin{tabular}{|l|l|l|}
\hline Name & Type & $\begin{array}{l}\text { Size - diameter } \mathbf{x} \\
\text { thickness (mm) }\end{array}$ \\
\hline E1 & Gel & $38 \times 1$ \\
\hline E2 & Gel & $24 \times 1$ \\
\hline E3 & Dry/Non-gel & $10 \times 2$ \\
\hline
\end{tabular}

Fig. 4. Three ECG electrodes (from-left-to-right): E2, E1, and E3 (Table 1)

\section{B. CBT Sensing Unit}

In the proposed smart sensor system, we have used a thermopile sensor that is a non-contact sensor for measuring core body temperature (CBT). Because tympanic temperature directly reflects the core temperature of the carotid artery [28], we have proposed an ear-bud design of infrared tympanic sensor that can continuously measure the temperature of the tympanic membrane. This design connects with an MCU which is the core controller of the whole smart sensor system to perform signal processing. The thermopile sensor (MLX90614) [22] gives a very sensitive information regarding the core temperature with 17 bit $\mathrm{ADC}$ resolutions, thus this could be in some cases $0.0034{ }^{\circ} \mathrm{C}$. Figure 5 illustrates the proposed design of CBT sensing unit and embedded thermopile sensor together.

(a)

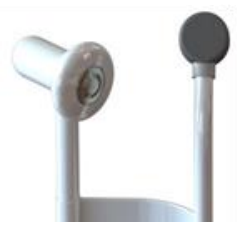

Fig. 5. CBT sensing components: (a) The proposed earphone-type infrared sensor, (b) infrared thermopile sensor

\section{PPG Sensing Unit}

The finger-worn PPG sensor consists of a transductor which initiates two LEDs and a photodiode detector. One of the LEDs emits red light (with a wavelength of $\lambda=660 \mathrm{~nm}$ ) and other LED performs an infrared light (with a wavelength of $\lambda=880 \mathrm{~nm}$ ). This technique is known as PPG (photoplethysmography) and the PPG sensor is based on the theory that the colour of blood range depends on the oxygen it contains. For instance, hemoglobin particularly reflects more red light and the PPG sensor detects oxygen saturation in the blood $\left(\mathrm{SpO}_{2}\right)$. For our test-bed application regarding PPG sensor unit, we measured both oxygen level in the blood $\left(\mathrm{SpO}_{2}\right)$ and heart rate together using Amperor Bluetooth Finger PPG sensor [24] which is shown in Figure 6. Proposed smart sensor system incorporates a Bluetooth connection to get heart rate and $\mathrm{SpO}_{2}$ from $\mathrm{PPG}$ sensor.

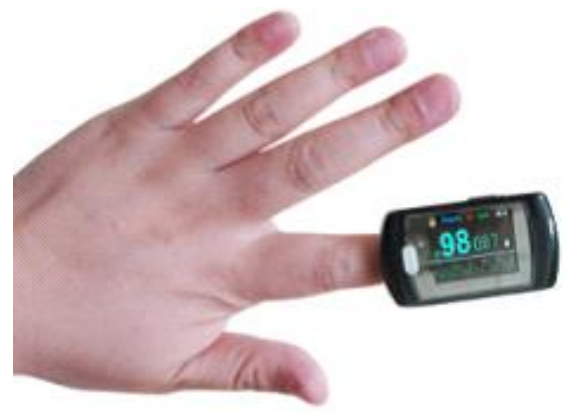

Fig. 6. Bluetooth finger PPG sensor

The analog signals from the sensors are conditioned at the wearable hardware unit to levels suitable for digitization and processing. Two stage amplification units were used with gain of 10 and 100 to avoid the noises overriding the ECG signals, which is achieved by an instrumentation amplifier, and a micro-power operational amplifier, respectively. The ECG signals are restricted in bandwidth of $0.5-100 \mathrm{~Hz}$ using a high pass and low pass filters after the first and second steps of amplification, respectively. The power line interference in the ECG signal is filtered using a $50 \mathrm{~Hz}$ notch filter to avoid loss of $50 \mathrm{~Hz}$ component of the ECG signals. The PPG sensor probe has an infrared source at $880 \mathrm{~nm}$ and the photodetector giving current output, which is converted to voltage by an instrumentation amplifier with gain 10 and using high and low pass filters between 0.5 and $20 \mathrm{~Hz}$. The CBT sensing block consists of a calibration circuit and a high gain amplifier with 10. The system runs from a lithium-battery (see in Figure7) which has a capacity of 500mAh, and lasts approximately 30 hours, depends on working with Bluetooth as it consumes a significant proportion of whole power assumption. The prototype ear-bud device was designed in SolidWorks and created using a 3D printer. The design is shown in Figure 5(a), measures $8.5 \mathrm{~cm}$ within cables and sits behind the patient's ear (mastoid area). The design was made using flexible unit to move around the ear and conform better to the ear so that it helps to secure the device.

\section{EXPERIMENTAL RESULTS}

In this section, the experimental tests and results were demonstrated individually from ECG, CBT and PPG sensors 
into a Matlab program. The impact of using different type of electrodes (gel and dry) was drawn for ECG monitoring system. Ear-lead ECG monitoring was compared to conventional chest-lead model and different scenarios were analysed as changing positions of ECG electrodes on the body to clearly see which scenario gives the best SNR and least noise. Furthermore, the integration of ECG, CBT and PPG sensors into wearable hardware unit and transmission the physiological data to the smartphone using Bluetooth radio were demonstrated in this section.

\section{A. ECG Experimental Results}

Figure 7 shows the experimental setup for ECG monitoring with wireless Android based smartphone. Figure 8a provides basic components of a typical ECG signal including various features (P, Q, R, S and T waves). A number of intervals can be measured and analyzed from an ECG recording. A normal ECG signal can give very important information regarding the heart status during a cardiac cycle. The time between the beginning of a particular point in a cardiac cycle and the beginning of another particular point in the next cardiac cycle is the interval between the beginning electrical responses of those particular points in the heart. For example, the heart rate can be measured by simply looking at R-R (beat-to-beat) interval, which indicates the time between two consecutive QRS complexes in an ECG recording. A sample ECG signal is shown in Figure $8 \mathrm{~b}$ including two successive beats (R-R intervals and P-QRS-T complexes). This test signal is used to compare with corrupted ECG signals from the sensor platform. The SNRs of ECG signals in Table 2 are calculated by differentiating between each signal in Figure 7 and this test ECG signal, respectively.



Fig. 7. Experimental device of Arduino based ECG measurement system with wireless smart phone monitoring

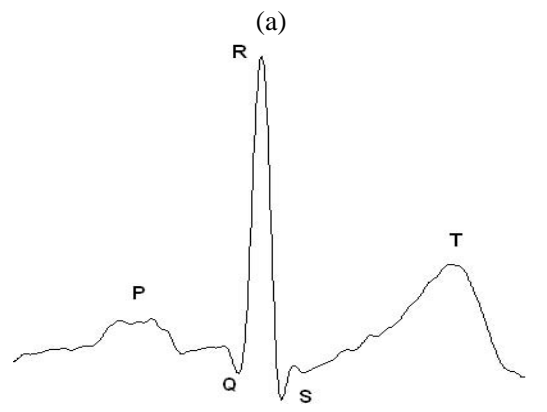

(b)

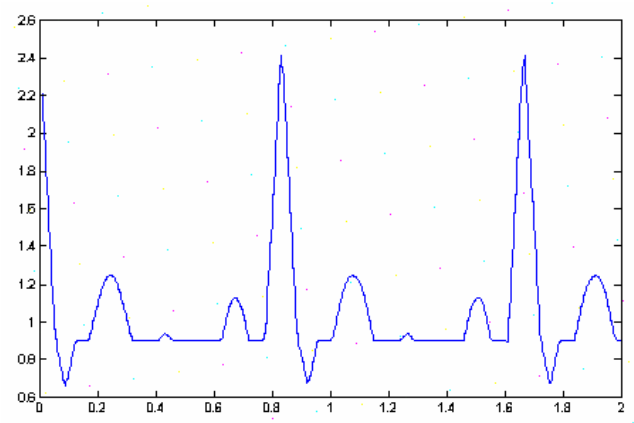

Fig. 8. ECG Signal Components: (a) A typical ECG signals including PQRS-T morphology; (b) Test signal to be compared with the signals obtained from our experiments

Figure 9 shows the placements and types of electrodes for ECG measurements reported in this paper. Different scenarios are drawn in the figure in order to see the important changes and what kind of challenges there are. Another aim is also to select the best unobtrusive scenario from the figure, according to ECG signal qualities, and SNR. As can be seen from the figure, the electrodes were placed on different locations on the body such as ear, chest and arm. Both the standard three gel $\mathrm{Ag} / \mathrm{AgCl}$ electrodes and dry electrodes with different positions were used to measure ECG signals.

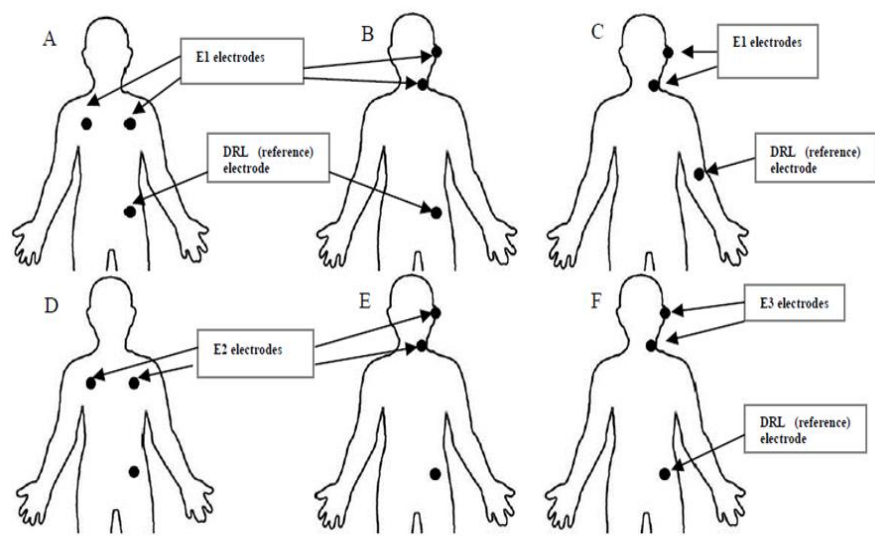

Fig. 9. The locations and type of the ECG electrodes for the measurements (Scenarios A-F)

All of ECG signals shown in Figure 10 were measured in lead I according to Einthoven's triangle [19] using ECG sampling module and rebuilt in Matlab without any software de-noise for further study. Power consumption is critical for such an application as this. The system runs from a lithiumbattery (see in Figure 4) which has a capacity of 500mAh, and lasts approximately 30 hours, this depends on the use of Bluetooth module as it consumes a significant proportion of the available power.

Figure 10 indicates ECG results from each electrode and each placement according to the scenarios which are shown in Figure 9. The electrodes with larger array size exhibits less noises due to larger skin-electrode contact area. Even the dry electrode provided ECG signals from behind-the-ear that was comparable to $\mathrm{Ag} / \mathrm{AgCl}$ electrode. The figures illustrate clear observations of the QRS complex and T-wave cardiac signs. 

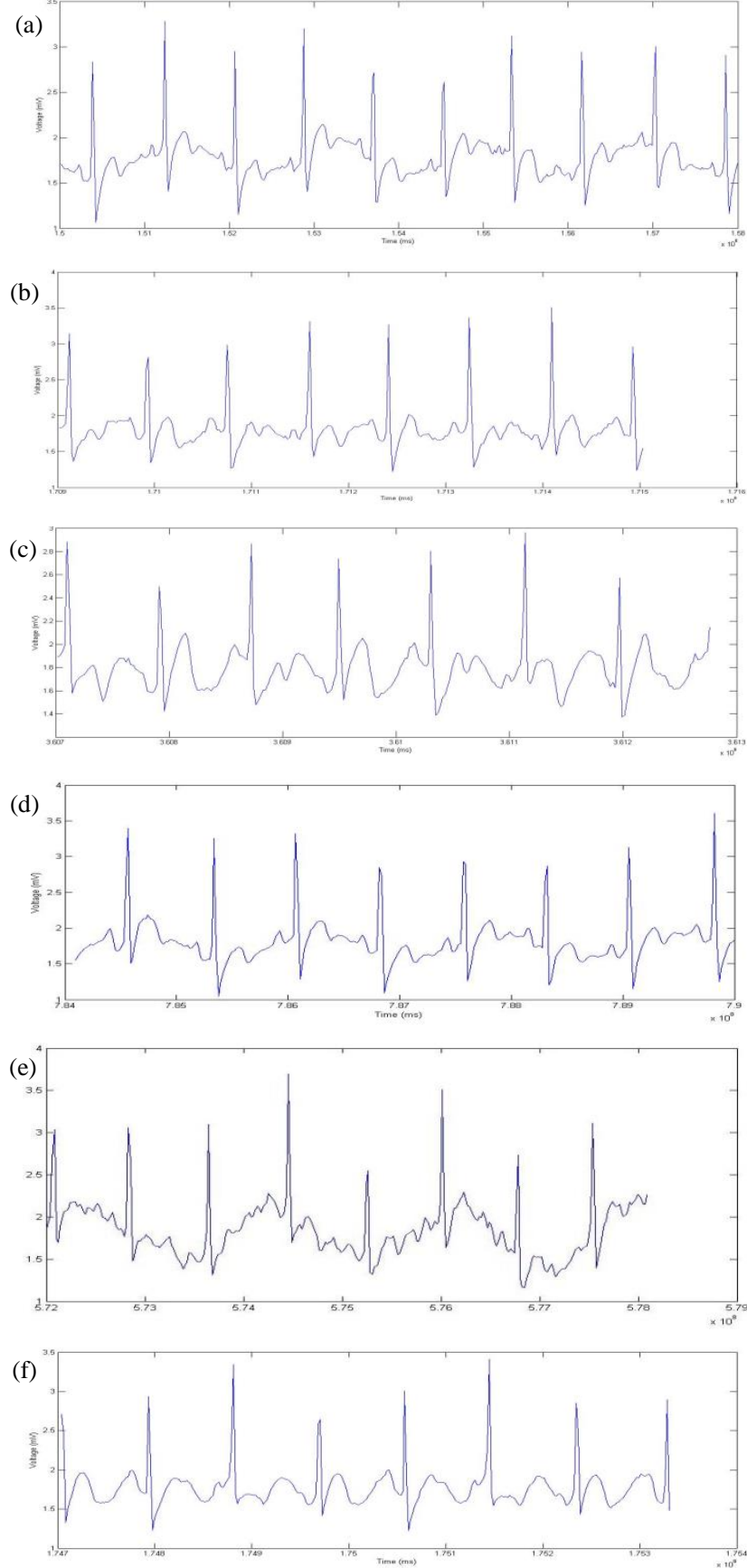

Fig. 10. The measured ECG signals at various locations as illustrated in Fig.9 (a) Scenario A; (b) Scenario B; (c) Scenario C; (d) Scenario D; (e) Scenario E; (f) Scenario F

As can be seen from Figure 9 and 10, E1 electrodes were applied in the measurements from (a) to (c). Figure 10(a) shows basic ECG measurement while two active electrodes were placed on the chest and one reference DRL (driven-rightleg) electrode was attached on the waist. This is a typical way of measuring ECG under the rule of 3-leads ECG recording [26]. Figure 10(b) indicates the ECG data taken from behindthe-ear and reference electrode was placed on the waist and Figure 10(c) shows the ECG signal for the same scenario as those in Figure 10(b) while reference electrode was placed on the arm instead of the waist. After that, we implemented E2 electrodes for measuring ECG and, Figure 10(d) illustrates ECG data taken from the chest and reference electrode was on the waist. Using the same type of electrodes, Figure 10(e) shows the ECG data coming from behind-the-ear and reference electrode was on the waist. Lastly, Figure 10(f) shows ECG signal using dry electrodes (E3 electrode) were placed behind the ear; and DRL electrode was on the waist. The received signal is accompanied with noise; however, $\mathrm{R}$ peaks (the most dominant feature in the ECG cycle) could be identified. Figure also illustrates that some distortions and fluctuations have occurred during ECG recording due to body movements and taking new position of ECG electrodes.

To further evaluate the performance of the electrodes, ECG signals were analyzed to calculate signal-to-noise ratio (SNR) using the following equation [14]:

$$
\mathrm{SNR}=20 \log \left(\mathrm{S} /\left(\mathrm{S}^{\prime}-\mathrm{S}\right)\right)
$$

where $\mathrm{S}$ is the filtered ECG signal with a frequency ranging from $0.5 \mathrm{~Hz}$ to $100 \mathrm{~Hz}$, and $\mathrm{S}^{\prime}$ is defined as ECG signal without filtering. Before calculation, the power line interference $(50 \mathrm{~Hz})$ was removed from both signals. Table 2 summarizes the SNR of 6 different ECG results which are represented in Figure 10 a-f, respectively.

TABLE II. SNR OF ECG Signals WITH DifFERENT EleCtrodes AND PLACEMENTS

\begin{tabular}{|l|l|l|}
\hline $\begin{array}{l}\text { Experiment } \\
\text { Scenario (see Fig 9) }\end{array}$ & SNR (dB) & Response Time (s) \\
\hline Scenario A & 25.21 & 0.85 \\
\hline Scenario B & 17.27 & 1.17 \\
\hline Scenario C & 12.95 & 1.80 \\
\hline Scenario D & 21.23 & 0.90 \\
\hline Scenario E & 15.34 & 1.25 \\
\hline Scenario F & 10.92 & $\sim 35$ \\
\hline
\end{tabular}

As seen from the results of Figure 10 and Table 2, it is obvious the ECG signals from the chest (Scenario A and Scenario D) are the best, in terms of the detections of various parts of the ECG $(\mathrm{P}, \mathrm{Q}, \mathrm{R}, \mathrm{S}$ and $\mathrm{T})$ and they are less noisy too. Moreover, when we compared Scenario A with Scenario D or Scenario B with Scenario E, figures clearly show that E1 electrodes provides better ECG waveforms in terms of noise than attached E2 electrodes due to larger skin-contact size. On the other hand, after waiting some time (longer response time as given Table 2) to remove unwanted distortions, the $\mathrm{P}$ wave and QRS complex can be identified in Scenario F, which is the most effective method because of the use of the dry electrodes.

As the work is aimed at behind the ear electrodes for the benefits already mentioned previously, it is clear from the results, behind-ear electrodes still produces comparable signals and hence usable for the purpose of convenient ECG detection. While analyzing the concept of Scenario F, thus, dry electrodes are chosen for detection of ECG waveforms and integration with other body sensors together.

Furthermore, we monitored ECG signals (including QRS complex) on an Android based smartphone including heart rate (HR) as shown in Figure 11(b). Android was used because of its widespread use and development and the software is portable for coding within mobile devices. Additionally, it allowed simplicity of integration with ECG sensors via 
Bluetooth. We also compared our ear-lead ECG results with recently proposed by $\mathrm{Da} \mathrm{He}$ [27] who worked to get ECG data using behind-the-ear device (Figure 11a).

(a)
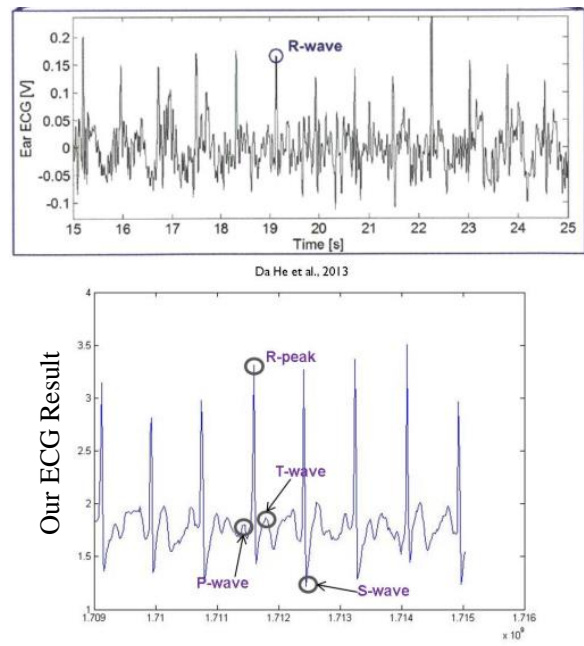

(b)

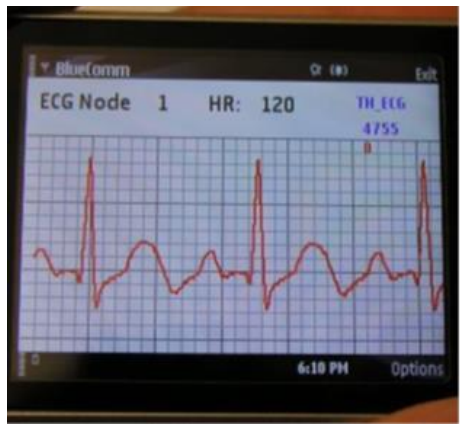

Fig. 11. (a) Compared our results with Da He's work on detecting ECG signals from the ear; (b) displaying our ECG data on the smartphone

Figure 11a shows a comparison of ECG results taken from the ear in Da He's work and ours. As can be seen, only Rpeaks were detected including various noises in his results; however, our results identify PQRS-complex and T-waves with less noise. The critical point here is that $\mathrm{Da} H e$ used two active electrodes because of the limited skin area near the ear, thus DRL (reference) electrode was omitted in his experiments.

The results reveal that the proposed gel electrode with reference electrode on the waist shows better signal quality and performance than other proposed electrodes. The table also indicates that once DRL electrodes placed on the waist, they have better SNR ratio than placed on the arm. Dry electrodes hold less signal quality than others, however, they still can be used to get ECG signal from inner ear area which is very useful for feasibility and interoperability issues. Response times of each proposed electrode shows that a similar advance with SNR ratio which means bigger size gel electrode gives a faster response.

\section{B. CBT Experimental Results}

Figure 12 shows the experimental device of CBT measurement and display of CBT results on the smartphone. Several analyses have been conducted on core body temperature during exercises to observe the changes in different environments (Figure 13). Researchers emphasize on analysis of core body temperature variations to diagnose or prevent serious diseases from heat illnesses. Heat illnesses can range from mild (e.g. heat rashes, heat cramps, etc.) to more severe health issues such as heat exhaustion and heat stroke caused by heat stress. Heat stress (hyperthermia - Temp > 37 ${ }^{\circ} \mathrm{C}$ ) is a condition when an individual is exposed to moderate to high temperatures from physical activity and some form of dehydration. Symptoms include blood pressure changes, increased heart rate and body temperature. The core body temperature is between $98.6^{\circ} \mathrm{F}\left(37^{\circ} \mathrm{C}\right)$ and $104^{\circ} \mathrm{F}\left(40^{\circ} \mathrm{C}\right)$. Symptoms include heavy sweating, rapid breathing, rapid yet weak heart rate and low blood pressure. The core body temperature exceeds $105^{\circ} \mathrm{F}\left(40.5^{\circ} \mathrm{C}\right)$, with symptoms including dizziness, lack of sweating, rapid and strong heart rate, high blood pressure [29]. Therefore, it is important to check out CBT regularly in healthcare for prevention of heat related diseases.


Fig. 12. (a) Experimental setup for Arduino based CBT measurement; (b) Monitoring of CBT results on the smartphone
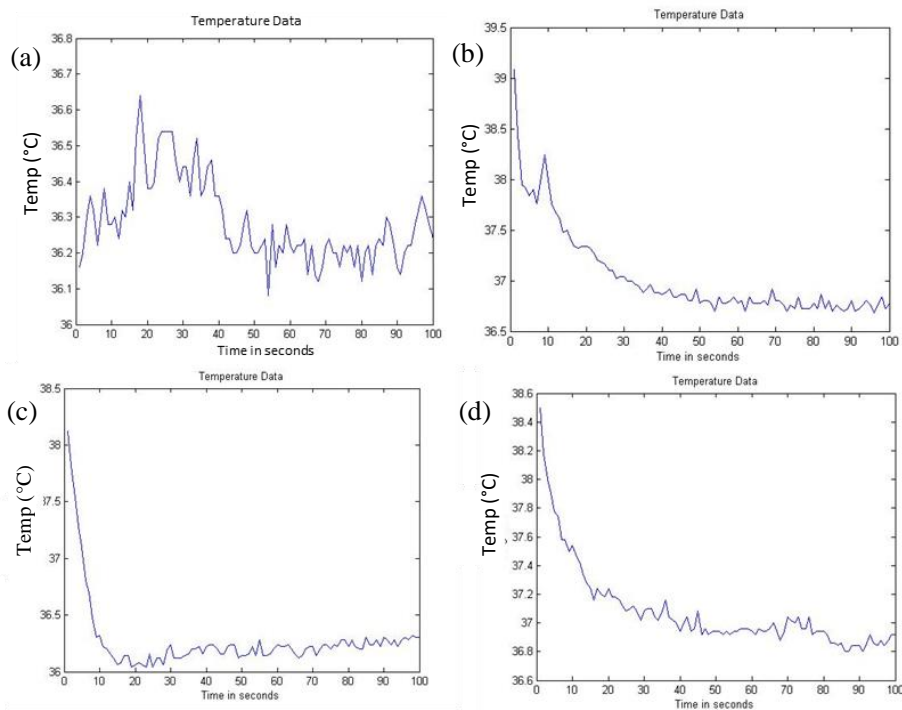

Fig. 13. (a) Raw CBT data taken from the ear; (b) CBT data just after exercise -5 min running; (c) CBT data after walking outside in cold weather; and (d) final temperature data after sitting 5 mins with a thick jacket 
Figure 13 shows four different results that were captured during the exercises in different ambient temperature values. Figure 10a indicates typical raw data of core body temperature which was taken from the ear without any exercise. It varies between $36{ }^{\circ} \mathrm{C}$ and $36.7{ }^{\circ} \mathrm{C}$ which is normal for a healthy person. We can certify that the core body temperature gets increased as the level of exercise gets harder, can be shown in Figure 13b. The temperature raised just over $37{ }^{\circ} \mathrm{C}$ after 5 minutes of running exercise. Figure $13 \mathrm{c}$ illustrates the changes of core body temperature against the change of ambient temperature. The subject was walking outside in cold and windy weather that had around $14{ }^{\circ} \mathrm{C}$ ambient temperature. Because of decreasing ambient temperature, the core body temperature gets decreased to nearly $36^{\circ} \mathrm{C}$ in this experiment. In Figure 13d, the core body temperature was captured when the subject was wearing a thick jacket in the office and ambient temperature was around $23{ }^{\circ} \mathrm{C}$. Thus, the core body temperature was elevated to almost $37.3{ }^{\circ} \mathrm{C}$ in some cases (Figure 13d).

\section{PPG Experimental Results}

Figure 14a shows a person's PPG waveform, heart rate (HR) and blood oxygenation together using the finger-clipped PPG sensor, which is connected to a smart sensor unit via Bluetooth. According to the results, values of oxygen saturation in the blood were around 95\%, and HR was around 93 beat per minute (bpm). Figure 14b demonstrates a PPG measurement on a 29 year-old subject with respect to $\mathrm{SpO}_{2}$ and HR during the exercise. As can be seen from Figure 14, there were variations regarding PPG data during a 15-minute exercise including sitting, standing and running. Particularly, in the period of running, there is a sharp increasing on HR values, however, blood oxygenation values were decreasing. These data were captured and displayed on a PC. After PPG data sent to the smart sensor unit, it was also displayed on the smartphone via Bluetooth connection.



(a)

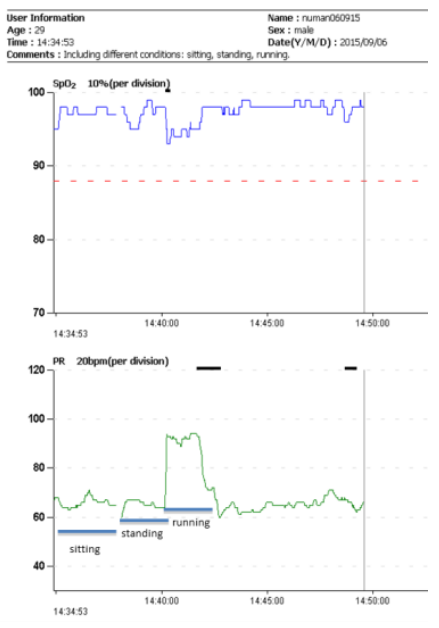

(b)
Fig. 14. (a) Measuring heart rate and SpO2 using Amperor Bluetooth PPG sensor; (b) a PPG measurement on a subject during exercise using the same device

\section{Proposed Sensor Integration}

After digitizing and conditioning the analog signals from each sensor, the suitable digital physiological data are collected at the data acquisition hardware unit in an appropriate way. The whole data after being acquired by Arduino is converted in the form of packets and wirelessly transmitted to an Android based mobile phone [11]. Figure 15 indicates ECG electrodes placement and appearance of general prototype of performed body sensors, including a subject wearing the smart sensor on the ear.
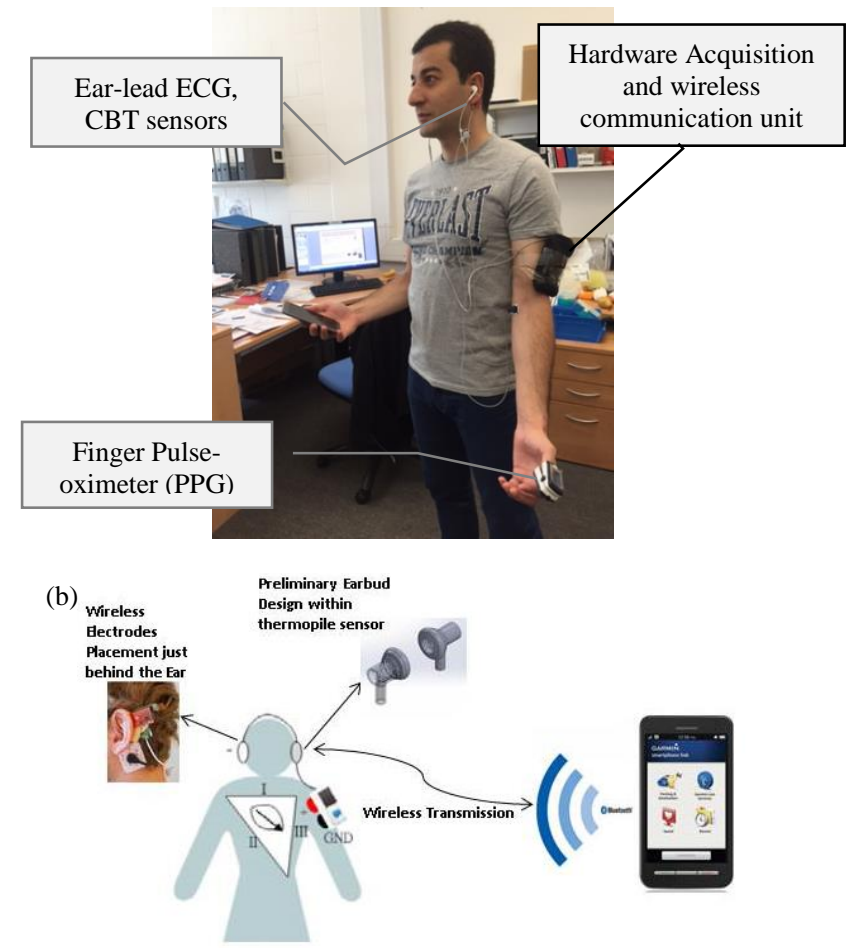

Fig. 15. (a) A typical set-up of the use of the proposed integrated wireless multiple sensors; CBT sensor was put into the earbud, and adhesive ECG sensors were attached onto the behind-the-ear and upper neck area; (b) prototype concept of Ear-lead wearable multiple sensor monitoring system

Figure 15 shows the developed experimental set-up and integration of protocol of sensors. Typically, both ECG adhesive $\mathrm{Ag}-\mathrm{AgCl}$ and dry electrodes were attached to the skin, respectively, to measure ECG data. Two active electrodes were applied to near the ear (behind-the-ear, and upper neck area), and one reference electrode was placed on the arm. CBT sensor was put into the earbud design which is also shown in Figure 15(b). PPG sensor was clipped on thumb finger to measure oxygen saturation level and heart rate. After all, the whole biological data coming from these three sensors are combined together into the Arduino hardware acquisition unit (Figure 15a) in suitable data packets. In the final step, the collected physiological data are transferred via Bluetooth module to an Android based smartphone and monitored using a newly developed app.

\section{E. Data Transmission and Bluetooth Connection}

Bluetooth is a very useful technology to communicate wirelessly in short-range applications such as exchanging data from short distance fixed mobile devices. With the characteristics of synchronization with other Bluetooth devices, the wireless body area network can be successfully applied. Our propose system uses HC-05 Bluetooth module that 
consists of different modes in its processing system. Figure 16 shows our Bluetooth serial module, which is paired to the smartphone.

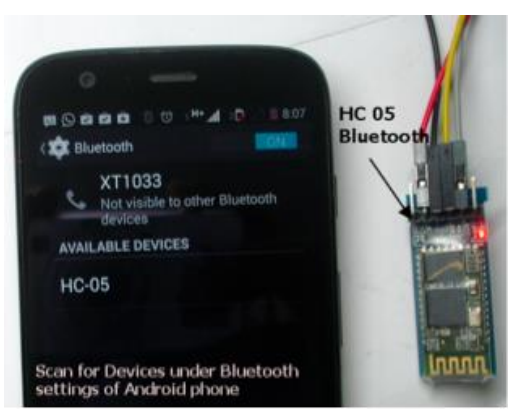

Fig. 16. The view of Bluetooth module while pairing with the smartphone

As mentioned, there are different channels (ECG, CBT and PPG) that need to be transmitted through Bluetooth communication. The communication module has the capability to transmit data over the Bluetooth with maximum baud rate 115,200 bps. For our system, Core body temperature (CBT) measurement operates at $20 \mathrm{~Hz}$ sampling frequency (16-bit samples); ECG sensor works at $250 \mathrm{~Hz}$ frequency range (16-bit samples) with 3 electrodes and PPG operating at a $30 \mathrm{~Hz}(16-$ bit samples) with two photodiodes. Hence, the total data rate will comprise from aggregating each measured signal's bit rates. The total minimum bandwidth of the system will then be:

Baud Rate $(\mathrm{BR})=$ bit rate of $(\mathrm{CBT}(\mathrm{kbps})+\mathrm{ECG}(\mathrm{kbps})+$ PPG (kbps))

$\mathrm{BR}=($ sampling frequency of $\mathrm{CBT}) *($ Nyquist-criteria $) *$ (sampling bit number) + (sampling frequency of ECG) * (Nyquist-criteria) * (sampling bit number) * (number of electrodes $)+($ sampling frequency of PPG) $*$ (Nyquist-criteria) * (sampling bit number) * (number of electrodes)

$$
\mathrm{BR}=20 * 2 \text { (Nyquist-criteria) } * 16+250 * 2 * 16 * 3+30
$$$$
* 2 * 16 * 2=0.64+24+1.92
$$

$$
\mathrm{BR}=26.56 \mathrm{kbps} \text {. }
$$

The above baud rate is much less than the overall Bluetooth transmission baud rate, whose transmission capacity is 115.2 kbps. Thus, the residual bandwidth would be enough to perform two-way handshaking and sending the bio potentials successfully.

\section{DISCUSSION AND CONCLUSION}

Personal healthcare applications bring a growth area for wearable health monitoring systems. Wearable diagnostics and therapeutic systems contribute intelligent medical monitoring devices, which provide real-time feedback to the patients or remote monitoring servers. Wearable physiological monitoring applications, by integrating of body sensors are significantly important for patients with chronic health conditions, especially chronic neurological disorders, cardiovascular diseases and strokes that are leading causes of mortality worldwide. However, a number of ongoing research efforts target on various technical issues that need to be resolved in order to have much more sensitive, reliable, secure, and powerefficient wireless personal area network suitable in particular for mobile healthcare applications. Existing technological advances in remote monitoring systems are sometimes incapable of performing real-time patient monitoring systems because of the inability of traditional wet electrodes to perform a long term monitoring, and lack of easy-for-use design. Moreover, new designs or perspectives need to be improved in conventional $\mathrm{Ag} / \mathrm{AgCl}$ electrodes for getting much more sensitive biological data due to lack of low-power microelectronics and miniaturization design in such applications. Wireless body sensors can be formed within a new technology perspective as well as the materials among mobile and wearable patient-monitoring devices to sense tiny biopotentials such as ECG and PPG from different locations on the body with very high reliability and accuracy. Otherwise, very noisy data will result using these conventional sensors, because such areas on the body (e.g. ear, upper neck) will be affected by motion artifacts.

The design and evaluation of an ear-lead multiple smart sensor system was presented in this paper. The system acquires different physiological information and continuously monitors an Android based smartphone, giving patients real-time control of data. This device includes non-intrusive sensors, specifically ECG, CBT and PPG with high accuracy. Moreover, we have also tested our Android based app, which combines the recording of all sensors together and displays ECG, CBT, and $\mathrm{SpO}_{2}$ biological data on the smartphone of an ambulatory user. We also attempted to observe the influence of sensor positioning on signal quality using various types of ECG electrodes. Furthermore, it facilitates the difficulties of wearables giving patients significantly less restriction by eliminating the need for adapting intrusive equipment or using a laptop to see the biological data. Our results clearly demonstrate the feasibility of the concepts and interoperability of the sensors and solutions to the key technological and scientific problems. Despite of making a significant progress in addressing many of the issues, there are still considerable issues that need to be improved. Future studies will take into account the evolution of conventional body sensors and new perspectives for the improvement of the design of the particular ECG electrodes to reduce noisy data due to motion artifacts. We will further investigate the aspects of an m-Health service that the role of the smartphone can be used as a wearable physiological monitoring system including providing a realtime feedback to the patients from a central server. Therefore, the smartphones can be seen as a gateway in mobile healthcare monitoring applications. Recent advances in research are leading to this realization by involving the benefits of nanotechnology in biomedical science such as the large surface area or high electrical conductivity values of novel nanomaterials.

In this paper an ECG electrode set-up is demonstrated to pick up ECG signals from behind-the-ear in contrast to tradition chest-based ECG measurements. The results obtained are very promising and detection of the components of the ECG signal (P, Q and $\mathrm{R}$ ) is highly possible. It was mentioned that the behind-the-ear ECG measurement is more userfriendly and gives extra convenience of using electrodes only when it is required (i.e.: no need to keep the electrodes attached all the time). A method of integrating ECG, PPG and co-body temperature using Arduino microcontroller and smart phone 
for processing and displaying the data is also presented. In the future, more experiments will be performed to design better electrodes with increased signal-to-noise ratio to obtain the results similar to the traditional ECG signs and to demonstrate the integrated systems under various operating conditions.

\section{ACKNOWLEDGMENT}

This research was supported by Ministry of National Education of Turkey and the research group of DocLab in Brunel University London.

\section{REFERENCES}

[1] Bifulco, P.; Cesarelli, M.; Fratini, A.; Ruffo, M.; Pasquariello, G.; Gargiulo, G. A Wearable Device for Recording of Biopotentials and Body Movements. In Proceedings of the IEEE International Symposium on Medical Measurements and Applications, Bari, Italy, 30-31 May 2011; pp. 469-472.

[2] Socio-economic impact of mHealth - An assessment report for the European Union. Available online: http://www.gsma.com/connectedliving/wpcontent/uploads/2013/06/Socio-economic_impact-ofmHealth_EU_14062013V2.pdf (accessed on 16 June 2015).

[3] Ren, Y.; Pazzi, R. W. N.; Boukerche, A. Monitoring patients via a secure and mobile healthcare system. Wireless Communications, IEEE, 2010, 17(1), 59-65.

[4] Lee, D. H.; Rabbi, A.; Choi, J.; Fazel-Rezai, R. Development of a mobile phone based e-health monitoring application. Int. J. of Adv. Comput. Sci. and App. 2012, 3(3), 38-43.

[5] Nemati, E.; Deen, M. J.; Mondal, T. A wireless wearable ECG sensor for long-term applications. Communications Magazine, IEEE, 2012, 50(1), 36-43.

[6] Sanches, J. M.; Pereira, B.; Paiva, T.; Headset Bluetooth and cell phone based continuous central body temperature measurement system. In Proceedings of the IEEE Engineering in Medicine and Biology Society, Buenos Aires, Argentina, August 31 - Sept 4, 2010, pp. 2975-2978.

[7] Jung, K. H.; Tran, V.; Gabrielian, V.; Nahapetian, A. Virtual cuff: multisensory non-intrusive blood pressure monitoring. In Proceedings of the 9th International Conference on Body Area Networks (BodyNets '14), London, UK, 29 September - 1 October 2014; pp. 175-178.

[8] Do Valle, B. G.; Cash S. S.; Sodini C. G. Wireless behind-the-ear eeg recording device with wireless interface to a mobile device (iphone/ipod touch). In Proceedings of 36th IEEE Engineering in Medicine and Biology Society (EMBS), Chicago, US, 26-30 August 2014; pp. 59525955.

[9] Song, W.; Yu, H.; Liang, C.; Wang, Q.; Shi, Y.; Body monitoring system design based on android smartphone. In Proceedings of 2012 World Congress on Information and Communication Technologies WICT 2012, Trivandrum, India, 30 October - 2 November 2012; pp. 1147-1151

[10] Boano, C. A.; Lasagni, M.; Romer, K.; Non-Invasive measurement of core body temperature in marathon runners. In Proceedings of the Body Sensor Networks 2013 (BSN '13), Cambridge, US, 6-9 May 2013; pp. $1-6$.

[11] Shen, T. W.; Hsiao, T.; Liu, Y. T.; He, T. Y. An ear-lead ECG based smart sensor system with voice biofeedback for daily activity monitoring. In TENCON 2008-2008 IEEE Region 10 Conference, Hyderabad, India, 19-21 November 2008; pp. 1-6.
[12] Hernandez, J.; McDuff, D. J.; Picard, R. W. BioPhone: Physiology Monitoring from Peripheral Smartphone Motions. In Proceedings of $37^{\text {th }}$ IEEE Engineering in Medicine and Biology Society (EMBS), Milano, Italy, 25-29 August 2015.

[13] Hii, P. C.; Chung, W. Y. A comprehensive ubiquitous healthcare solution on an Android ${ }^{\mathrm{TM}}$ mobile device. Sensors, 2011, 11(7), 67996815.

[14] Tranquillo, J. V. (2013). Biomedical signals and systems. Synthesis Lectures on Biomedical Engineering, 8(3), 1-233.

[15] Wahl, F.; Freund, M.; Amft, O. WISEglass: Smart eyeglasses recognising context. In Proceedings of the 10th International Conference on Body Area Networks (BodyNets '15), Sydney, Australia, 28-30 September 2015.

[16] Poh, M. Z.; Kim, K.; Goessling, A.; Swenson, N.; Picard, R. Cardiovascular monitoring using earphones and a mobile device. IEEE Pervasive Computing, 2012, (4), 18-26.

[17] Morón, M. J.; Luque, R.; Casilari, E. On the capability of smartphones to perform as communication gateways in medical wireless personal area networks. Sensors, 2014, 14(1), 575-594.

[18] Lee, B. G.; Lee, B. L; Chung, W. Y. Smartwatch-based driver alertness monitoring with wearable motion and physiological sensor. In Proceedings of $37^{\text {th }}$ IEEE Engineering in Medicine and Biology Society (EMBS), Milano, Italy, 25-29 August 2015.

[19] J. Malmivuo and R. Plonsey. Bioelectromagnetism - Principles and Applications of Bioelectric and Biomagnetic Fields . Oxford University Press, New York, 1995.

[20] Lin, Y. H.; Lin, C. F.; You, H. Z. A driver's physiological monitoring system based on a wearable PPG sensor and a smartphone. SecurityEnriched Urban Computing and Smart Grid, 2011, 223, 326-335.

[21] ArduinoUNO. Available online: https://www.arduino.cc/en/Main/ArduinoBoardUno (accessed on 16 May 2015).

[22] Infrared Thermometer - MLX90614. Available online: https://www.sparkfun.com/products/9570 (accessed on 18 May 2015).

[23] ECG Acquisition System. Available online: https://www.cookinghacks.com/electrocardiogram-sensor-ecg-ehealth-medical (accessed on 12 May 2015).

[24] Pulse Oximeter Module. Available online: http://www.contecmed.com/ (accessed on 19 May 2015).

[25] HC Bluetooth Transceiver Module. Available online: http://www.gearbest.com/ (accessed on 21 May 2015).

[26] De Luna, A. B.; Batchvarov, V. N.; Malik, M. The Morphology of the Electrocardiogram. 2006. Available online: https://www.blackwellpublishing.com/content/BPL_Images/Content_sto re/Sample_chapter/9781405126953/9781405126953_4_001.pdf (accessed on 10 June 2015).

[27] He, D. D.; Winokur, E. S.; Sodini, C. G. An Ear-Worn Vital Signs Monitor. Biomedical Engineering, IEEE Transactions, 2015, 62(11), 2547-2552.

[28] Bock, M.; Hohlfeld, U.; Von Engeln, K.; Meier, P. A.; Motsch, J.; Tasman, A. J. The accuracy of a new infrared ear thermometer in patients undergoing cardiac surgery. Canadian Journal of Anesthesia, 2005, 52(10), 1083-1087.

[29] Heat Illness: MedlinePlus. Available online: https://www.nlm.nih.gov/medlineplus/heatillness.html (accessed on 22 June 2015). 